Journal of Applied Fluid Mechanics, Vol. 7, No. 2, pp. 263-273, 2014.

Available online at www.jafmonline.net, ISSN 1735-3572, EISSN 1735-3645.

DOI: $10.36884 / j a f m .7 .02 .19421$

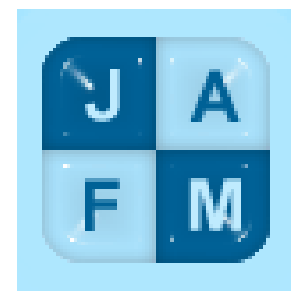

\title{
Cell Model for Slow Viscous Flow past Spherical Particles with Surfactant Layer Coating
}

\author{
S. Datta ${ }^{1}$ and S. Raturi ${ }^{2+}$ \\ ${ }^{1,2}$ Department of Mathematics and Astronomy, Lucknow University, Lucknow, India-226007 \\ ${ }^{\dagger}$ Corresponding Author Email: shwetaraturi05@gmail.com
}

(Received January 25, 2013; accepted July 9, 2013)

\begin{abstract}
Creeping flow through a swarm of spherical particles, where each particle consists of a solid core covered by a liquid shell coated with monomolecular layer of surfactant layer, is studied using the cell model technique. The analytical solution of the problem for four models: Happel's, Kuwabara's, Kvashin's and Cunningham's (usually referred to as Mehta-Morse's) is derived. The drag force acting on each particle in the cell is evaluated for the four models. In limiting cases the drag force reduces to earlier analytical results. Results are discussed and presented in graphical forms.
\end{abstract}

Keywords: Drag force, Interface, Surface shear viscosity, Surface dilatational viscosity.

\section{1- INTRODUCTION}

The presence of surfactant, at gas/liquid, liquid/liquid and air/liquid interface, has a profound effect on the stress balance at the interface associated to the bulk fluid. When surfactants concentrate in an adsorbed monolayer at a surface or interface, the property of the interface needs a modification. Forces, now acting on the interface, are surface tension gradients and the viscous resistance to shear and dilatation. While in the bulk liquids, the dilatational viscosity of fluids can typically be neglected and only a Newtonian shear viscosity needs to be considered, two fundamental deformations take place at the interface and a viscosity can be associated with each of them. When the surfactant monomolecular layer of fluid is present at the interface, the boundary conditions at the free surface or at an interface get altered due to the presence of surface layer parameters $\kappa$ (surface dilatational viscosity), $\varepsilon$ (surface shear viscosity) and $\sigma$ (surface tension). The surfactants are insoluble in both bulk phases, and the interface obeys a linear rheological model given by Boussinesq (1913) and generalized by Scriven (1960) constitutive law. Surfactants have a remarkable ability to influence the properties of interfaces, and thereby have an impact on industrial

processes and products; this has led to ubiquitous development of surfactant science resulting in a lot of published literature on this growing subject.
The motion of liquid drops and gas bubbles in the presence of surfactants is of major importance because of its significance in a variety of applications, such as nuclear physics, meteorology, biotechnology, medical science and chemical engineering. The earliest investigations of the motion of a liquid drop in another immiscible liquid were carried out by Rybezynski (1911) and independently by Hadmard (1911). Later on the dynamics of fluid interfaces were investigated by Lamb (1932), Reid (1960), Miller and Scriven (1968), Ramabhadran et al., (1976) and Prosperetti (1977, 1980). The motion of a spherical bubble rising steadily in dilute surfactant solutions has been investigated by Harper (1974, 1982 and 1988). Levan (1981) extended the study of Levan and Newman (1976) of droplet motion in the presence of surfactants to treat a droplet with a Newtonian interface. Sadhal and Johnson (1983) derived an exact solution for creeping flow past a bubble or drop with a stagnant cap of surfactant film at the rear. The effect of surfactants on drop deformation and breakup was examined by Stone and Leal (1990). Further, Stone (1994) studied the dynamics of drop deformation and breakup in viscous fluid. Warszynski, et al. (1996) demonstrated the non-equilibrium distribution of surfactant over the surface of a bubble after it had risen different distances. Numerical methods have been developed by Ryskin and Leal (1983, 1984a, 1984b), McLaughlin (1996) to solve for fluid flow around a rising bubble. The effects of insoluble surfactants and the drop deformation on the hydrodynamic interactions and on the rheology of dilute emulsions are the subjects of investigation by Li and Pozrikidis (1997), Blawzdziewicz et al. (1999), and Blawzdziewicz et al. (2000). Recently, 
Li and Mao (2001) have investigated numerically the effect of surfactant on the motion of the drop at intermediate Reynolds number. Danov (2001) have studied a liquid droplet in a shear flow in the presence of surfactant and had taken out the analytical solution of the problem. Datta and Pandya (2002) extended the study of Taylor and Acrivos (1964); to investigate the deformation of the spherical drop, where an absorbed monomolecular surfactant film of fluid is present on the surface of the drop. Gupta and Deo (2013) have studied the axisymmetric flow of a micropolar fluid over a sphere coated with a thin fluid film.

Fluid flow through an assemblage of particles arises in many multiphase systems common in chemical and engineering processes and has been generating scientific interest for more than fifty years. However, the mathematical formulation of such problems is a cumbersome task as one has to analyze the complex interaction between numerous particles in order to get information about the flow field. The cell model technique Happel and Brenner (1983) involves the concept that random assemblage of particles can be divided into a number of identical cells; one particle enveloped by each cell was used to overcome this problem. Thus the problem is reduced to consideration of a single particle and its bounding envelope and the interaction effect is accounted by the application of suitably chosen boundary conditions at the envelope of the cell.

Uchida (1949) proposed a cell model to study the creeping flow in an infinite cubic assemblage. He singled out a particle from the swarm and assumed it to be confined within a cubic cell acting as a fluid envelope. The advantage of the model is that a cubic envelope is space filling however, a major drawback of the model is that of difference in outer and inner geometry. Later on Happel $(1958,59)$ remodeled Uchida's model by taking both the outer and inner surfaces as spheres/cylinders. Happel assumed vanishing of shearing stress on the cell signifying no friction between the particles of swarms due to interaction. On the other hand Kuwabara (1959) assumed nil vorticity on the cell surface i.e. the flow is of potential kind. Both the formulations give almost same results but in Kuwabara's case there is slight exchange of mechanical energy between cell and environment. The mechanical power given by the sphere to the fluid is not all consumed by viscous dissipation in the fluid layer instead a small part is given to the environment. On the contrary no such exchange takes place in Happel's case.

Mehta-Morse (1975)/Cunningham (1910) acknowledged the importance of unknown velocity as a boundary condition on the cell surface. Thus, they used the uniform velocity condition on hypothetical cell to investigate flow through charged membranes. This assumption signifies the homogeneity of flow at the cell. Kvashnin (1979) used the symmetry condition for velocity by assuming that tangential component of velocity approaches extreme value on the cell surface along radial direction.

This paper concerns a brief review of the four boundary conditions on the cell surface, having monomolecular layer of surfactant at the interface and to find an expression for drag taking into account all the effects due to the presence of surfactants. Stokes equation is used to study the flow inside the spherical particle and outside it. We have used the cell model technique to study the flow. The importance of cell model technique is that we can put a suitable boundary condition on the cell surface which takes into account the perturbation effect of other particles of the assemblage on the particle concerned. As boundary conditions, continuity of tangential velocity and discontinuity of normal velocity and tangential stress at the spherical shell and vanishing of velocity on the solid spherical core are employed. On the hypothetical surface, continuity of radial component of velocity and the boundary conditions of all the four different cell models (Happel, Kuwabara, Kvashin and Mehta - Morse) are used. The drag force experienced by shell enclosed in a cell is evaluated. In limiting cases, earlier results reported by Happel, Kuwabara, Kvashin and Mehta Morse, have been deduced. Variation of drag for different parameters is presented in graphical form and is compared for different models.

\section{MATHEMATICAL FORMULATION OF THE PROBLEM}

Here, we consider a concentrated system of identical particles each of radius $a$, consisting of a solid core of radius $\bar{R}$ covered by a liquid shell of thickness $\bar{\delta}$ coated with a surfactant layer $\bar{r}=a$ with surface shear viscosity $\bar{\varepsilon}$ and surface dilatational viscosity $\bar{\kappa}$. The system is placed in a uniform flow with ambient velocity $\mathrm{U} \overrightarrow{e_{z}}$ along the z-axis. The problem will be investigated using the cell method; thus, it is assumed that each particle is located inside a concentric spherical cell of radius $b$ as shown in Fig.1. The radius $b$ of the cell is chosen in such a way that

$$
\frac{4}{3} \pi b^{3}=\frac{1}{n}
$$

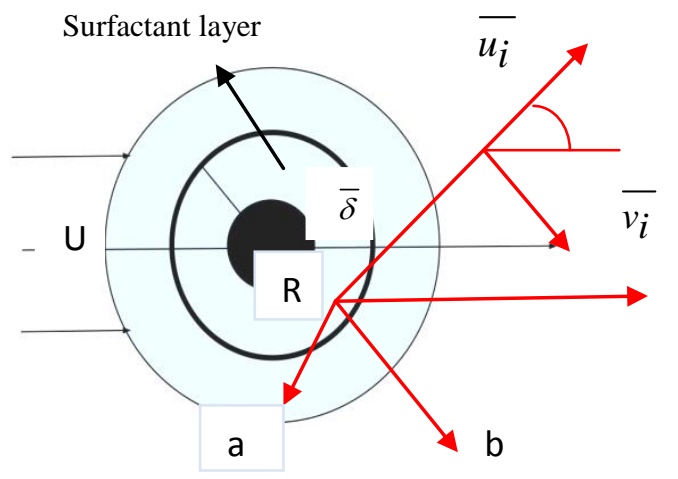

Fig. 1. Spherical cell of radius $b$ with a solid particle of radius $\bar{R}$ covered by a liquid shell of thickness $\bar{\delta}$ coated with a monomolecular surfactant layer $\bar{r}=a$.

Let us introduce a spherical co-ordinate system $(\bar{r}, \theta, \phi)$ with the origin located at the particle center and the line 
S. Datta and S. Raturi. / JAFM, Vol. 7, No. 2, pp. 263-273, 2014.

$\theta=0$ as the axis of symmetry, in the direction of the free stream velocity $U$ approaching the system. Due to axis-symmetry all the physical qua quantities are independent of $\phi$. Thus we have $\partial / \partial \phi=0$. The flows inside the shell ( $\bar{R} \leq \bar{r} \leq a)$ and outside the shell ( $a \leq \bar{r} \leq b$ ) are governed by radial and transverse components of Stokes equation and the equation of continuity

$$
\begin{aligned}
\frac{1}{\mu} \frac{\partial \bar{p}_{i}}{\partial \bar{r}}= & \frac{\partial^{2} \bar{u}_{i}}{\partial \bar{r}}+\frac{2}{\bar{r}} \frac{\partial \overline{u_{i}}}{\partial \bar{r}}+\frac{1}{\bar{r}^{2}} \frac{\partial^{2} \bar{u}_{i}}{\partial \theta^{2}}+\frac{\cot \theta}{\bar{r}^{2}} \frac{\partial \bar{u}_{i}}{\partial \theta} \\
& -\frac{2 \bar{u}_{i}}{\bar{r}^{2}}-\frac{2}{\bar{r}} \frac{\partial \bar{v}_{i}}{\partial \theta}-\frac{2 \bar{v}_{i} \cot \theta}{\bar{r}^{2}} \\
\frac{1}{\mu \bar{r}} \frac{\partial \bar{p}_{i}}{\partial \theta}= & \frac{\partial^{2} \bar{v}_{i}}{\partial-2}+\frac{2}{r} \frac{\partial \overline{v_{i}}}{\partial \bar{r}}+\frac{1}{\bar{r}^{2}} \frac{\partial^{2} \bar{v}_{i}}{\partial \theta^{2}}+\frac{\cot \theta}{\bar{r}^{2}} \frac{\partial \overline{v_{i}}}{\partial \theta} \\
& +\frac{2}{\bar{r}} \frac{\partial \bar{u}_{i}}{\partial \theta}-\frac{\overline{v_{i}} \cos e c^{2} \theta}{\bar{r}^{2}} \\
\frac{\partial}{\partial \bar{r}}\left(\bar{r}^{2} u_{i}\right) & +\frac{\bar{r}}{\sin \theta} \frac{\partial}{\partial \theta}\left(\bar{v}_{i} \sin \theta\right)=0
\end{aligned}
$$

where $i=1$ refers to the physical quantities in the interior of the shell $(\bar{R} \leq \bar{r} \leq a), i=2$ refers to the physical quantities in the exterior of the shell $(a \leq \bar{r} \leq b)$; also $\bar{u}_{i}$ and $\bar{v}_{i}$ are components of velocities in the direction of $\bar{r}$ and $\theta$. Further $\bar{p}_{i}$ and $\mu_{i}$ are respectively the pressure and fluid viscosity.

The following boundary conditions are used to analyze the flow in the two regions. No-slip and no penetration conditions are imposed on the surface of solid sphere

$$
\overline{v_{1}}=0 \quad \text { and } \quad \overline{u_{1}}=0 \quad \text { at } \quad \bar{r}=\bar{R}
$$

On the interface $\bar{r}=a$, we assume no-penetration and continuity of tangential velocity

$$
\left.\begin{array}{l}
\overline{u_{i}}=0 \\
\overline{v_{1}}=\overline{v_{2}}
\end{array}\right\} \quad \text { at } \bar{r}=a
$$

Next boundary conditions at the interface as derived from the work of Scriven (1960) are discontinuity of tangential stress and normal stress. The discontinuity of normal stress determines the deformation of the surfactant layer boundary shape but since this is being neglected, only the discontinuity of tangential stress is taken into account here.
Taking the surface tension as constant, Scriven's (1960) boundary condition at $\bar{r}=a$ reduces to

$$
\begin{aligned}
& \bar{\tau}_{r \theta(1)}-\bar{\tau}_{r \theta(2)}=2 \bar{\varepsilon} \frac{\bar{v}}{\overline{r^{2}}}+ \\
& (\bar{\kappa}+\bar{\varepsilon}) \frac{\partial}{\partial \theta}\left(\frac{1}{\overline{r^{2}} \sin \theta} \frac{\partial}{\partial \theta}(\bar{r} \bar{v} \sin \theta)\right) \\
& \bar{\tau}_{r \theta(i)}=\mu_{i}\left(\bar{r} \frac{\partial}{\partial \bar{r}}\left(\frac{\bar{v}_{i}}{\bar{r}}\right)+\frac{1}{\bar{r}} \frac{\partial \bar{u}_{i}}{\partial \theta}\right)
\end{aligned}
$$

Boundary conditions on the hypothetical cell $\bar{r}=b$ reduce a complex problem to a simpler one. There are four frequently used boundary conditions, viz. Happel's, Kuvabara, Kvashnin and Mehta-Morse (Cunningham). All four models assume continuity of the radial component of the liquid on the hypothetical cell surface $\bar{r}=b$

$$
\overline{u_{2}}=U \cos \theta
$$

An additional condition is used in each of the above mentioned models. Happel's (1958) model assumes vanishing of the shear stress at hypothetical cell surface, $\bar{r}=b$

$$
\frac{1}{r} \frac{\partial \overline{u_{2}}}{\partial \theta}+\frac{\partial \overline{v_{2}}}{\partial \bar{r}}-\frac{\overline{v_{2}}}{\bar{r}}=0
$$

Kuwabara's (1959) model assumes vanishing of vorticity at hypothetical cell surface, $\bar{r}=b$

$$
-\frac{1}{\bar{r}} \frac{\partial \overline{u_{2}}}{\partial \theta}+\frac{\partial \overline{v_{2}}}{\partial \bar{r}}+\frac{\overline{v_{2}}}{\bar{r}}=0
$$

Kvashin's (1979) model assumes symmetry condition at hypothetical cell surface, $\bar{r}=b$

$$
\frac{\partial \overline{v_{2}}}{\partial \bar{r}}=0
$$

Mehta-Morse's (1975) model assumes homogeneity at hypothetical cell surface, $\bar{r}=b$

$$
\overline{v_{2}}=-U \sin \theta
$$

which one of the four models is the most appropriate remains to be answered. In the present paper we investigate all the four models and compare the results.

It will be convenient to render the problem in nondimensional form by using the following dimensionless variables: 
S. Datta and S. Raturi. / JAFM, Vol. 7, No. 2, pp. 263-273, 2014.

$$
\begin{aligned}
& \frac{b}{a}=\frac{1}{\gamma}, r=\frac{\bar{r}}{a}, \delta=\frac{\bar{\delta}}{a}, R=\frac{\bar{R}}{a}=1-\delta, u_{i}=\frac{\overline{u_{i}}}{U} \\
& v_{i}=\frac{\overline{v_{i}}}{U}, m=\frac{\mu_{1}}{\mu_{2}}, \kappa=\frac{\bar{\kappa}}{\mu_{2} a}, \varepsilon=\frac{\bar{\varepsilon}}{\mu_{2} a}, p_{i}=\frac{\bar{p}_{i}}{\mu_{i} U / a}
\end{aligned}
$$

$\delta$ being the thickness of the shell and $\gamma^{3}=\left(\frac{a}{b}\right)^{3}$, being the volume fraction of the particles.

As the flow is axis-symmetric we introduce the Stokes stream function $\psi_{i}(r, \theta)$ satisfying the equation of continuity (3) on taking

$$
u_{i}=\frac{1}{r^{2} \sin \theta} \frac{\partial \psi_{i}}{\partial \theta}, v_{i}=-\frac{1}{r \sin \theta} \frac{\partial \psi_{i}}{\partial r}
$$

where $\psi_{1}(r, \theta)$ and $\psi_{2}(r, \theta)$ correspond respectively to regions inside the shell ( $R \leq r \leq 1)$ and outside the shell ( $1 \leq R \leq 1 / \gamma)$. Eliminating pressure $p_{i}$ between Eq. (1) and Eq. (2) and making use of Eq. (12) and Eq. (13), we get

$$
E^{4} \psi_{i}=0
$$

Where $E^{2}$ denotes the Stokes stream function operator given by

$$
\begin{aligned}
& E^{2}=\frac{\partial}{\partial r^{2}}+\frac{1-\zeta^{2}}{r^{2}} \frac{\partial^{2}}{\partial \zeta^{2}} \\
& \zeta=\cos \theta
\end{aligned}
$$

\section{SOLUTION OF THE PROBLEM}

In the case of axi-symmetric incompressible creeping flow the required regular solutions of the Stokes Eq. (14) is expressible as

$$
\psi_{i}(r, \theta)=\frac{\sin ^{2} \theta}{2}\left[A_{(i)} \frac{1}{r}+B_{(i)} r+C_{(i)} r^{2}+D_{(i)} r^{4}\right]
$$

The boundary conditions, in non-dimensional form given by Eqs. (4)- (11), reduce to:

$$
\begin{aligned}
& \psi_{1}=0 \\
& \frac{\partial \psi_{1}}{\partial r}=0
\end{aligned}
$$$$
\text { at } r=1-\delta
$$

$$
\left.\begin{array}{l}
\psi_{i}=0 \\
\frac{\partial \psi_{1}}{\partial r}=\frac{\partial \psi_{2}}{\partial r} \\
\tau_{\mathrm{r} \theta(2)}-\mathrm{m} \tau_{\mathrm{r} \theta(1)}=\frac{2 \varepsilon}{\sin \theta} \frac{\partial \psi}{\partial r}+ \\
(\kappa+\varepsilon) \frac{\partial}{\partial \theta}\left\{\frac{1}{\sin \theta} \frac{\partial}{\partial \theta}\left(\frac{\partial \psi}{\partial r}\right)\right\}
\end{array}\right\} \text { at } \mathrm{r}=1
$$

$$
\left.\frac{\partial \psi_{2}}{\partial \theta}=r^{2} \sin \theta \cos \theta \quad\right\} \text { at } r=1 / \gamma
$$

Happel condition:

$$
\begin{aligned}
& -\frac{\cot \theta}{r^{2}} \frac{\partial \psi_{2}}{\partial \theta}+\frac{1}{r^{2}} \frac{\partial^{2} \psi_{2}}{\partial \theta^{2}} \\
& -\frac{\partial^{2} \psi_{2}}{\partial r^{2}}+\frac{2}{r} \frac{\partial \psi_{2}}{\partial r}=0
\end{aligned} \quad \text { at } r=1 / \gamma
$$

Kuwabara condition:

$$
\begin{array}{cc}
\frac{\cot \theta}{r^{2}} & \frac{\partial \psi_{2}}{\partial \theta}-\frac{1}{r^{2}} \frac{\partial^{2} \psi_{2}}{\partial \theta^{2}} \\
-\frac{\partial^{2} \psi_{2}}{\partial r^{2}} & =0
\end{array}
$$

Kvashnin condition:

$$
\frac{1}{r} \frac{\partial \psi_{2}}{\partial r}-\frac{\partial^{2} \psi_{2}}{\partial r^{2}}=0 \quad \text { at } r=1 / \gamma
$$

Mehta-Morse condition:

$$
\frac{\partial \psi_{2}}{\partial r}=r \sin ^{2} \theta \quad \text { at } r=1 / \gamma
$$

Using the above boundary conditions given by Eqs. (17) (20) in Eq. (16) we get the values of the respective eight constants $A_{1}, A_{2}, B_{1}, B_{2}, C_{1}, C_{2}, D_{1}$ and $D_{2}$ for the four different models. Since these values are long, we refrain from presenting the values here.

\section{EVALUATION OF DRAG FORCE}

Evaluation of drag force is important in the applications of the flow problem we are investigating. Drag on the sphere 
S. Datta and S. Raturi. / JAFM, Vol. 7, No. 2, pp. 263-273, 2014.

is the force exerted on it by the moving fluid. It is evaluated by summing up the contributions of normal and tangential stress components to the force along the flow direction. Let $\overline{\tau_{r r}}$ and $\overline{\tau_{r \theta}}$ be the normal and tangential stress components on the surface of the particle, a sphere of unit radius $a$. Now hydrodynamic drag is given by

$$
\bar{D}=2 \pi a^{2} \int_{o}^{\pi}\left[\overline{\tau_{r r}} \cos \theta-\overline{\tau_{r \theta}} \sin \theta\right]_{\overline{\mathrm{r}}=a} \sin \theta d \theta
$$

where in the present case, we have relevant stress

$$
\begin{aligned}
& \text { components } \quad \bar{\tau}_{r \theta(2)}=\mu_{2}\left(\bar{r} \frac{\partial}{\partial \bar{r}}\left(\frac{\bar{v}_{2}}{\bar{r}}\right)+\frac{1}{\bar{r}} \frac{\partial \bar{u}_{2}}{\partial \theta}\right) \\
& \text { and } \bar{\tau}_{r r(2)}=-\overline{p_{2}}+2 \mu_{2} \frac{\partial \bar{u}_{2}}{\partial r}
\end{aligned}
$$

Using Eq. (1) and Eq. (2) we calculate the value of pressure distribution $\bar{p}_{2}$ and the value of $\bar{u}_{2}$ and $\bar{v}_{2}$ can be calculated with the help of Eq. (13) and Eq. (16). Substituting these values we get:

$$
\begin{aligned}
& \bar{\tau}_{r r(2)}=\frac{\mu_{2} U}{a}\left(-3\left(2 A_{2}+B_{2}+2 D_{2}\right) \cos \theta\right) \\
& \bar{\tau}_{r \theta(2)}=\frac{\mu_{2} U}{a}\left(-3\left(A_{2}+A_{8}\right) \sin \theta\right)
\end{aligned}
$$

Inserting the values of $\bar{\tau}_{r r(2)}$ and $\bar{\tau}_{r \theta(2)}$ in Eq. (21) and integrating, we get

$$
\bar{D}=-4 \pi a \mu_{2} U B_{2}
$$

Inserting the value of $B_{2}$ we get, on non-dimensionalizing with $a \mu_{2} U$, the nondimensional drag force, $\mathrm{D}$ for the four cases as follows.

Happel's model:

$$
\begin{aligned}
& D=-\left[4 \pi \left\{3 m\left(3+2 \gamma^{5}\right)(-2+\delta)(5+\delta(-5+2 \delta))\right.\right. \\
& \left.\left.-\delta(15+\delta(-15+4 \delta))\left(3(1+\kappa)+\gamma^{5}(-3+2 \kappa)\right)\right\}\right] / \\
& {\left[( - 1 + \gamma ) ^ { 2 } \left\{3 m\left(-2-\gamma+\gamma^{3}+2 \gamma^{4}\right)(-2+\delta)\right.\right.} \\
& (5+\delta(-5+2 \delta))-\delta(15+\delta(-15+4 \delta))(-3 \\
& \left.\left.\left.\left(1+\gamma+\gamma^{2}+\gamma^{3}+\gamma^{4}\right)+\left(-2-\gamma+\gamma^{3}+2 \gamma^{4}\right) \kappa\right)\right\}\right]
\end{aligned}
$$

$$
\begin{gathered}
D=-[60 \pi\{3 m(-2+\delta)(5+\delta(-5+2 \delta))-\delta(15+ \\
\delta(15+4 \delta))(1+\kappa)\}] /\left[(-1+\gamma)^{2}\{6 m(-1+\gamma)\right. \\
(5+\gamma(6+\gamma(3+\gamma)))(-2+\delta)(5+\delta(-5+2 \delta)) \\
-\delta(15+\delta(-15+4 \delta))(-3(5+\gamma(4+\gamma(3+\gamma(2+ \\
\gamma))))+2(-1+\gamma)(5+\gamma(6+\gamma(3+\gamma))) \kappa)\}]
\end{gathered}
$$

Kvashin's model:

$$
\begin{gathered}
D=-\left[1 2 \pi \left\{6 m\left(4+\gamma^{5}\right)(-2+\delta)(5+\delta(-5+2 \delta))\right.\right. \\
\left.-\delta(15+\delta(-15+4 \delta))\left(8(1+\kappa)+\gamma^{5}(-3+2 \kappa)\right\}\right] / \\
{\left[(-1+\gamma)^{2}\{3 m(-1+\gamma)(16+\gamma(21+\gamma(15+8 \gamma)))\right.} \\
(-2+\delta)(5+\delta(-5+2 \delta))-\delta(15+\delta(-15+4 \delta)) \\
(-3(8+\gamma(7+\gamma(6+\gamma(5+4 \gamma))))+(-1+\gamma) \\
(16+\gamma(21+\gamma(15+8 \gamma))) \kappa)\}]
\end{gathered}
$$

Mehta Morse's model:

$$
\begin{gathered}
D=-\left[1 2 \pi \left\{6 m\left(-1+\gamma^{5}\right)(-2+\delta)(5+\delta(-5+2 \delta))-\delta\right.\right. \\
\left.\left.(15+\delta(-15+4 \delta))\left(-2(1+\kappa)+\gamma^{5}(-3+2 \kappa)\right)\right\}\right] / \\
{\left[(-1+\gamma)^{3}\{3 m(-1+\gamma)(4+\gamma(7+4 \gamma))(-2+\delta)\right.} \\
(5+\delta(-5+2 \delta))-\delta(15+\delta(-15+4 \delta))(-3 \\
\left.\left.\left.\left(1+\gamma+\gamma^{2}+\gamma^{3}+\gamma^{4}\right)+\left(-2-\gamma+\gamma^{3}+2 \gamma^{4}\right) \kappa\right)\right\}\right]
\end{gathered}
$$

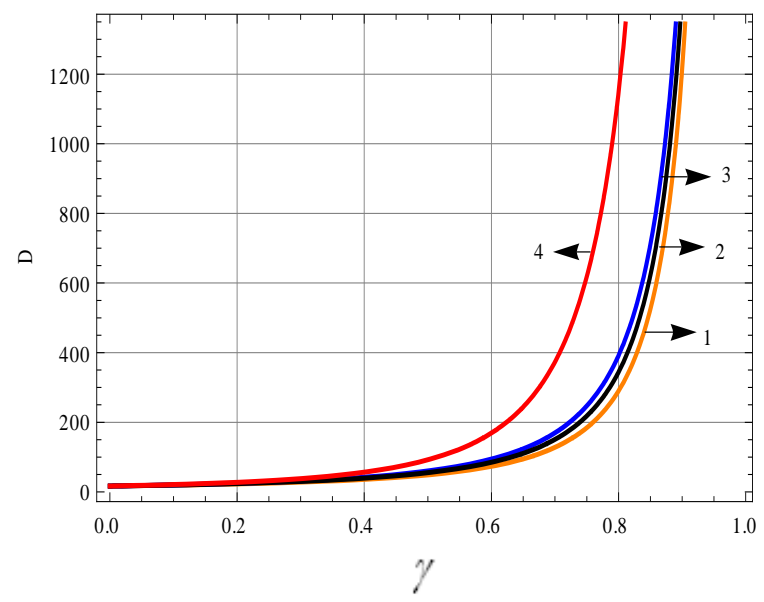

Fig. 2. Variation of drag force with $\gamma(\text { volume fraction of particles })^{1 / 3}$ for different models Happel (1), Kvashin(2), Kuwabara(3) and Mehta Morse (4) at $m=1, \delta=0.5$ and $\kappa=0$.

Kuwabara's model: 


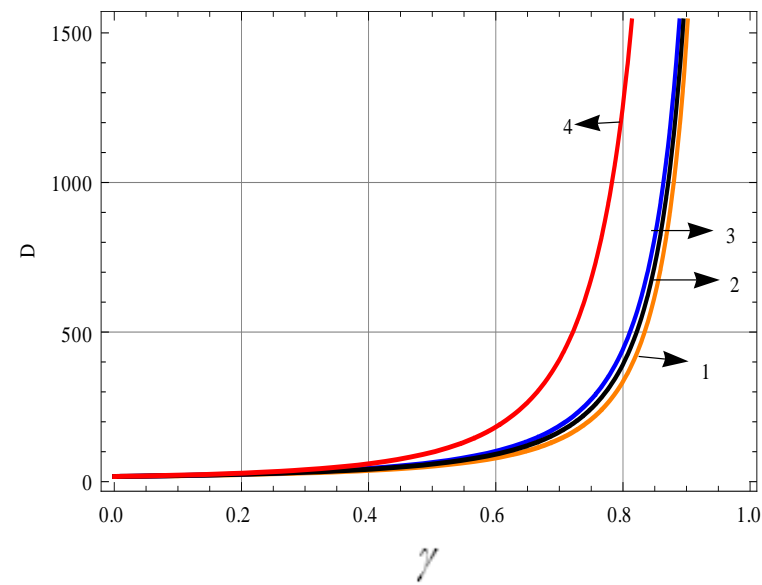

Fig. 3. Variation of drag force with $\gamma(\text { volume fraction of particles })^{1 / 3}$ for different models Happel (1), Kvashin (2), Kuwabara (3) and Mehta Morse (4) at $m=1, \delta=0.5$ and $\kappa=1$.

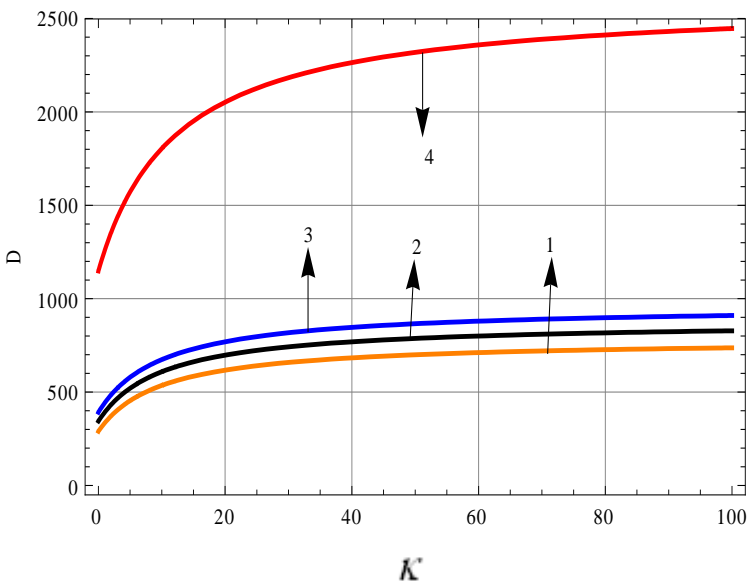

Fig. 4. Variation of drag force with $\kappa$ (surface dilatational viscosity) for different models Happel (1), Kvashin (2), Kuwabara (3) and Mehta Morse (4) at $\gamma=0.8, \delta=0.5$ and $m=1$.

Figure 2 shows that as $\gamma$ increases drag force increases and the rate of increment is greater at higher values of $\gamma$. The drag force coincides for the three models (Happel, Kuwabara, and Kvashin). However, Mehta-Morse model shows a deviation from the above three models. This model reports a greater drag force at higher volume fraction when compared with the other three models. Figure 3 shows the variation of drag force with $\gamma$ for all four models with $\kappa=1, m=1$ and $\delta=0.5$ and is of same pattern as Fig 2 . Figure 3 along with Fig. 2 shows that surface dilatational viscosity $\kappa$ increases the drag force. Figure 4 supports this fact as we see that the drag force increases with surface dilatational viscosity $\kappa$.

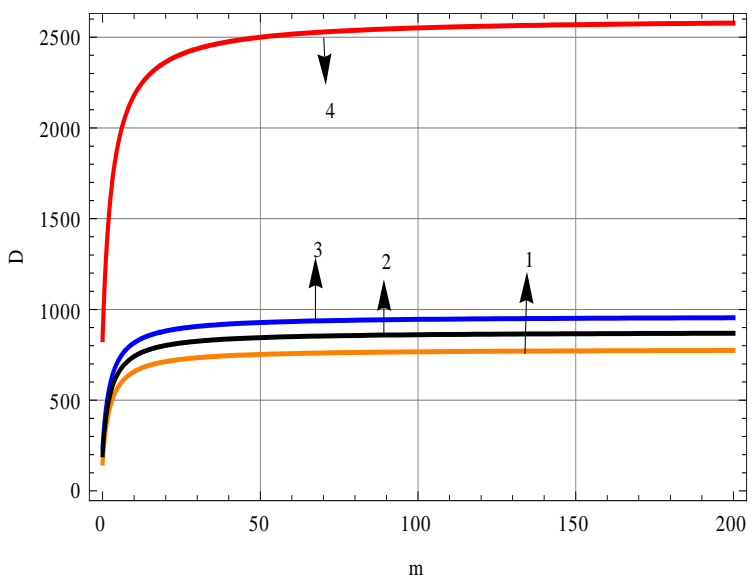

Fig. 5. Variation of drag force with $\mathrm{m}$ for different models Happel (1), Kvashin (2), Kuwabara (3) and Mehta Morse (4) at $\gamma=0.8, \delta=0.5$ and $\kappa=1$.

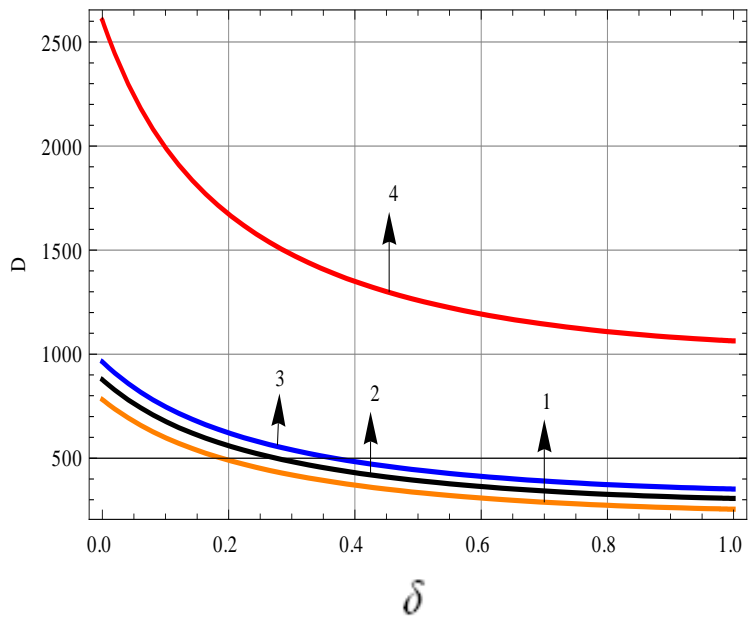

Fig. 6. Variation of drag force with $\delta$ (shell thickness) for different models Happel (1), Kvashin (2), Kuwabara (3) and Mehta Morse (4) at $\gamma=0.8, \kappa=1$ and $m=1$.

Figure 5 shows that as the inner viscosity increases thereby increasing $m$, the drag force increases; this increase is sharp in the beginning and then tends to a constant value for large inner viscosity as then the flow inside the shell practically vanishes.

Figure 6 shows that as the shell thickness $\delta$ (characterizing the thickness of the shell) increases drag force decreases for all four models. This decrease is greatest for Mehta-Morse model.

\subsection{Limiting cases and some known results}

1. When $\mathrm{m} \rightarrow \infty$ (i.e., solid particle), the expression

Happel's model: 
S. Datta and S. Raturi. / JAFM, Vol. 7, No. 2, pp. 263-273, 2014.

$$
D=\frac{4 \pi\left(3+2 \gamma^{5}\right)}{\left(2-3 \gamma+3 \gamma^{5}-2 \gamma^{6}\right)},
$$

the well known result of Happel and Brenner (1983) Kuwabara's model:

$$
D=\frac{30 \pi}{\left(5-9 \gamma+5 \gamma^{3}-\gamma^{6}\right)}
$$

the well known result of Kuwabara (1959)

Kvashin's model:

$$
D=\frac{24 \pi\left(4+\gamma^{5}\right)}{(1-\gamma)^{3}\left(16+21 \gamma+15 \gamma^{2}+8 \gamma^{3}\right)}
$$

the well known result of Kvashin (1979)

Mehta Morse's model:

$$
D=\frac{24 \pi\left(1+\gamma+\gamma^{2}+\gamma^{3}+\gamma^{4}\right)}{(1-\gamma)^{3}\left(4+7 \gamma+4 \gamma^{2}\right)}
$$

the well known result of Mehta Morse (1975).

Figure7 shows the variation of drag force, D with volume fraction of particles, $\gamma^{3}$ for the above four models

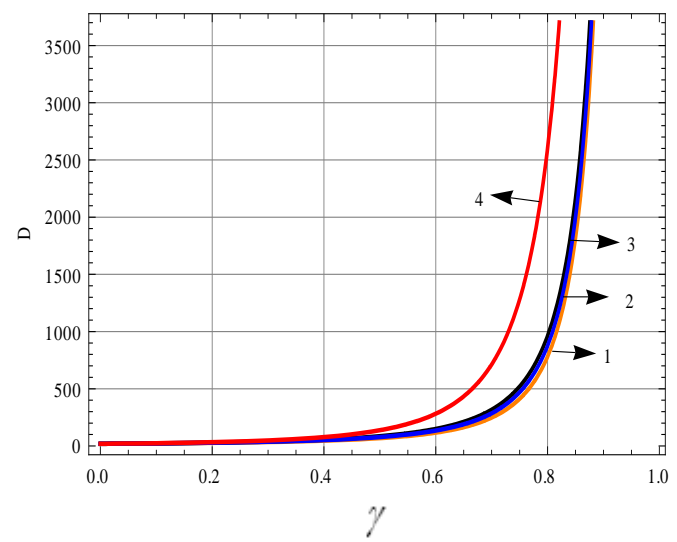

Fig. 7. Variation of drag force with $\gamma(\text { volume fraction of particle })^{1 / 3}$ for different models Happel (1), Kvashin (2), Kuwabara (3) and Mehta Morse (4).

2. When $\delta=1$, the shell will reduce to a fluid sphere covered by surfactant layer (i.e., absence of the solid core in the particle centre). Expressions for the drag D, for different models take the following form:

Happel's model:

$$
\begin{gathered}
D=-4 \pi\left\{m\left(9+6 \gamma^{5}\right)+6(1+\kappa)+\gamma^{5}(-6+4 \kappa)\right\} /\{-6+ \\
m\left(-6+9 \gamma-9 \gamma^{5}+6 \gamma^{6}\right)-6 \gamma^{5}(-1+\kappa)-4 \kappa \\
\left.+6 \gamma(1+\kappa)+\gamma^{6}(-6+4 \kappa)\right\}
\end{gathered}
$$

Kuwabara's model:

$$
\begin{aligned}
& D=-30 \pi(2+3 m+2 \kappa) /\left\{3 m \left(-5+9 \gamma-5 \gamma^{3}\right.\right. \\
& \left.\left.+\gamma^{6}\right)-10 \gamma^{3} \kappa+18 \gamma(1+\kappa)+\gamma^{6}(-3+2 \kappa)-5(3+2 \kappa)\right\}
\end{aligned}
$$

Kvashin's model:

$$
\begin{gathered}
D=-24 \pi\left\{3 m\left(4+\gamma^{5}\right)+8(1+\kappa)+\gamma^{5}(-3+2 \kappa)\right\} /\{3 m \\
(-1+\gamma)^{3}\left(16+21 \gamma+15 \gamma^{2}+8 \gamma^{3}\right)+2\left(-9 \gamma^{5}(-1+\kappa)\right. \\
\left.\left.-10 \gamma^{3} \kappa+27 \gamma(1+\kappa)+4 \gamma^{6}(-3+2 \kappa)-8(3+2 \kappa)\right)\right\}
\end{gathered}
$$

Mehta Morse's model:

$$
\begin{aligned}
D= & -24 \pi\left\{3 m\left(-1+\gamma^{5}\right)-2(1+\kappa)+\gamma^{5}(-3+2 \kappa)\right\} \\
& /\left[( - 1 + \gamma ) ^ { 3 } \left\{3 m\left(-4-3 \gamma+3 \gamma^{2}+4 \gamma^{3}\right)+2(-6+\right.\right. \\
& \left.\left.\left.3 \gamma^{2}(-3+\kappa)-4 \kappa-3 \gamma(3+\kappa)+\gamma^{3}(-6+4 \kappa)\right)\right\}\right]
\end{aligned}
$$

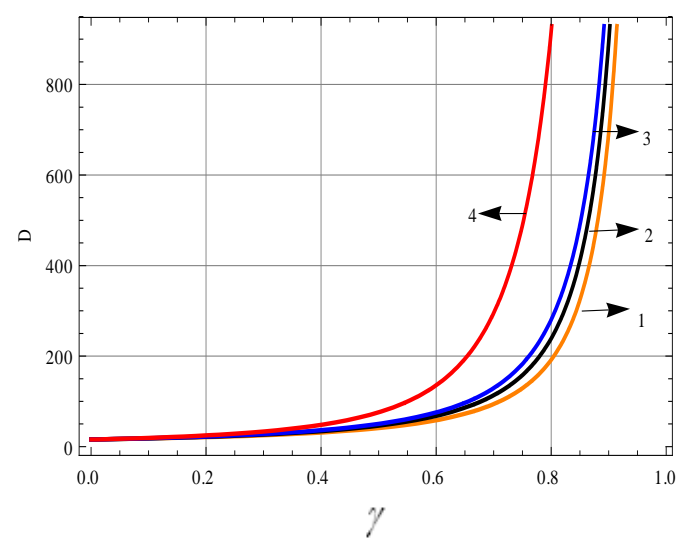

Fig. 8. Variation of drag force with $\gamma(\text { volume fraction of particles })^{1 / 3}$ for different models Happel (1), Kvashin(2), Kuwabara(3) and Mehta Morse (4) at $m=1, \delta=1$ and $\kappa=0$.

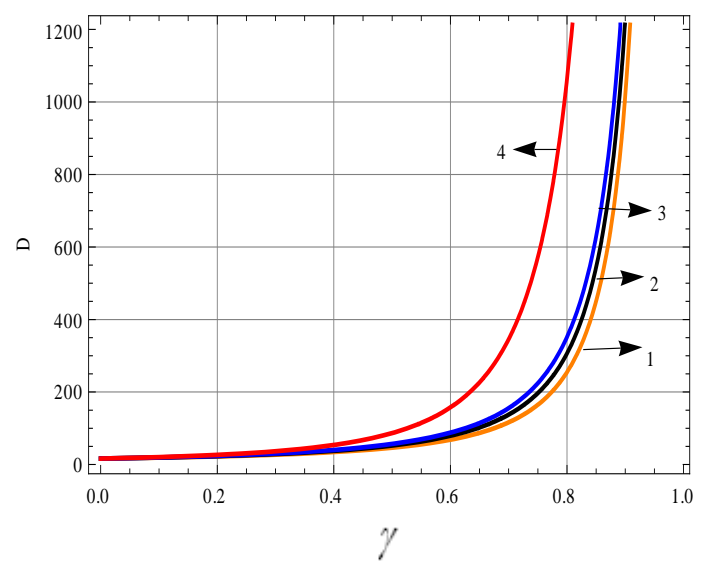

Fig. 9. Variation of drag force with $\gamma(\text { volume fraction of particles })^{1 / 3}$ for different models Happel (1), Kvashin(2), Kuwabara(3) and Mehta Morse (4) at $m=1, \delta=1$ and $\kappa=1$. 
Figure 8 and Fig. 9 shows the variation of drag force with $\gamma$ at $\delta=1$ (i.e., solid core is absent) and for $\kappa=0$ and $\kappa=1$ respectively for four different models. Comparison of the ordinate values of Fig. 8 and Fig. 9 with Fig. 2 and Fig. 3 shows that due to the absent of solid core drag force reduces and all the other variations are same as was reported in Fig. 2 and Fig. 3.

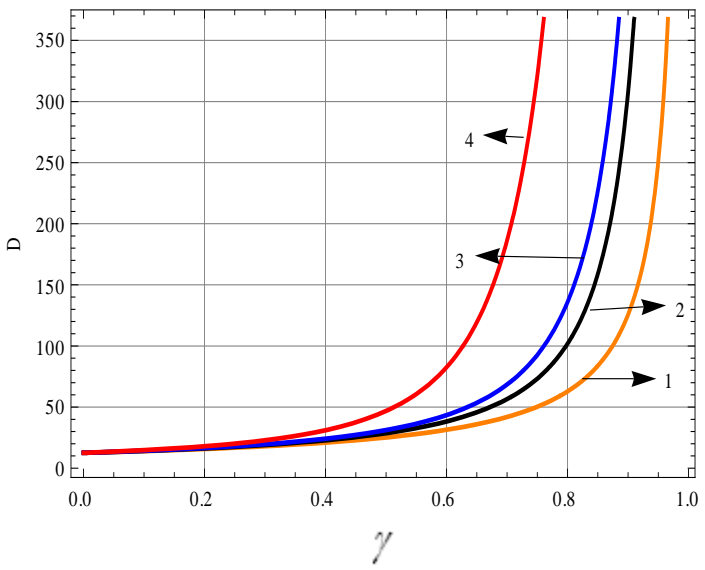

Fig. 10. Variation of drag force with $\gamma(\text { volume fraction of particles })^{1 / 3}$ for different models Happel (1), Kvashin (2), Kuwabara (3) and Mehta Morse (4) at $m=0, \delta=1$ and $\kappa=0$.

Figure 10 and Fig.11 shows the variation of drag force when $m \rightarrow 0$ i.e., the ideal fluid, for four different models. Comparison of Fig. 10 and Fig. 11 with Fig. 8 and Fig. 9 shows that the numerical value of drag force has decreased further the three models (Happel, Kuwabara and Kvashin) now show some difference in their values that was seen to be almost absent in Fig. 2, Fig. 3, Fig. 8 and Fig. 9.

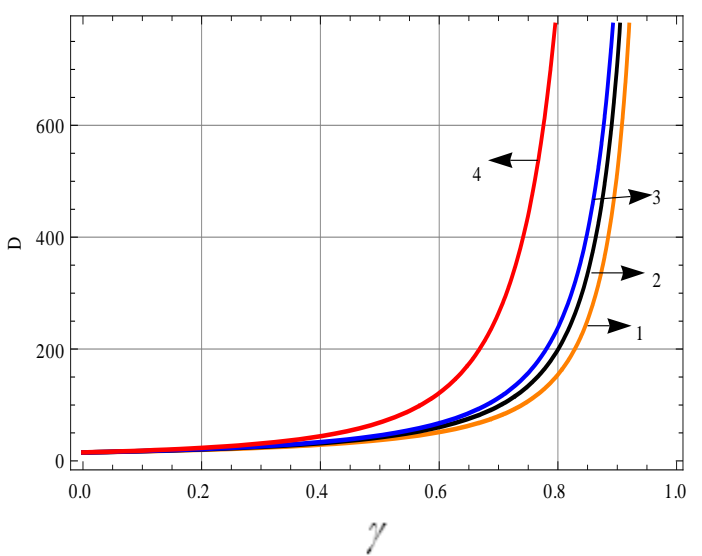

Fig. 11. Variation of drag force with $\gamma(\text { volume fraction of particles })^{1 / 3}$ for different models Happel (1), Kvashin (2), Kuwabara(3) and Mehta Morse (4) at $m=0, \delta=1$ and $\kappa=1$.
3. When $\gamma=0$, i.e. (b large), solid core is covered by a liquid shell coated with a surfactant layer placed in a uniform flow. For all the four models we get naturally the indentical result for drag

$$
\begin{gathered}
D=-12 \pi\{-6 m(-2+\delta)(5+\delta(-5+2 \delta))+2 \delta(15+ \\
\delta(-15+4 \delta))(1+\kappa)\} /\{12 m(-2+\delta)(5+\delta(-5+2 \delta)) \\
+\delta(15+\delta(-15+4 \delta))(-6-4 \kappa)\}
\end{gathered}
$$$$
\text { In the above case if } \delta=1
$$$$
D=\frac{6 \pi(2+3 m+2 \kappa)}{(3+3 m+2 \kappa)}
$$

The hydrodynamic drag force exerted on a solid particle of radius $\bar{R}$ that moves with a velocity $U$ is obtained by putting $m=1, \delta=0$ in Eq. (25). In dimensional form it is the classical Stokes drag formula: $\overline{D_{S}}=6 \pi \mu_{2} \bar{R} U$

Providing in non-dimensional form

$D_{S}=6 \pi$

With fluid shell coated with surfactant layer on the solid sphere surface, the hydrodynamic drag force

exerted will increase. The effective increase $\overline{L_{h}}$ (Happel 1983), of the particle radius to account for the corresponding increase in the hydrodynamic drag force is given by the relation

$$
\bar{D}=6 \pi \mu_{2}\left(\bar{R}+\overline{L_{h}}\right) \bar{U}
$$

Defining $\Omega=\frac{\bar{D}}{\overline{D_{S}}}=\frac{D}{D_{S}}$ (Masliyah et al., 1987; Vasin et al., 2008), as the ratio of the hydrodynamic drag force and Stokes force $\overline{D_{S}}=6 \pi \mu_{2} \bar{R} U$, we have

$$
L_{h}=\Omega-R
$$

The expression for the ratio of the hydrodynamic drag force, $\Omega$ takes the following form by substituting the values of $D$ and $D_{S}$ :

$$
\begin{gathered}
\Omega=-2\{-6 m(-2+\delta)(5+\delta(-5+2 \delta))+2 \delta(15+\delta(-15+ \\
4 \delta))(1+\kappa)\} /\{12 m(-2+\delta)(5+\delta(-5+2 \delta))+\delta(15+ \\
\delta(-15+4 \delta))(-6-4 \kappa)\}
\end{gathered}
$$

From Eq. (27) we can conclude that:

a) If $\delta=0$ (i.e. when fluid shell is absent and $\mathrm{R}=1$ ); then we have $\Omega=1$ confirming that the hydrodynamic drag force is equal to the Stokes force. 
b) If $K \rightarrow \infty$, then $\Omega=1$ and so the Stokes force is recovered again.

c) If $m \rightarrow \infty$, then $\Omega=1$ and so the Stokes force is recovered again.

d) If $\delta=1, R=0$; then $\Omega$ reduces to :

$$
\Omega=\frac{2+3 m+2 \kappa}{3+3 m+2 \kappa}
$$

Further if $m=1$, then the expression for the dimensionless hydrodynamic drag force ratio reduces to:

$$
\Omega=\frac{5+2 \kappa}{6+2 \kappa}
$$

e) If $m=0$, then we have

$$
\Omega=\frac{2(1+\kappa)}{3+2 \kappa}
$$

The variation of the dimensionless drag force ratio $\Omega$ as given by Eq. (27) with the parameter $\kappa$ at different $\delta$ is presented in Fig. 12. The decrease of drag force ratio is seen to be more pronounced at lower values of $\kappa$ and then it increases to its asymptotic value 1 as observed in case (b). The increase of the thickness of the fluid shell, represented by $\delta$, contributes to the decrease of drag ratio

The dimensionless drag force ratio $\Omega$, as given by Eq. (27) is plotted against the parameter $m$ at different values of the dimensionless thickness $\delta$ of the fluid shell in Fig. 13. All plots starts from an identical point determined by Eq. (28). In the above figure this point is $\Omega=0.67$. The value of this identical point increases when $\kappa$ increases. As viscosity ratio $m$ increases, the drag force increases and $\Omega \rightarrow 1$ as $m \rightarrow \infty$. This maximum value of $\Omega$ is reached earlier at low thickness of the shell $\delta$. Next if $m=$ 1 , the expression for the dimensionless hydrodynamic drag force ratio reduces

$$
\begin{aligned}
\Omega= & \left\{60+30 \delta(-2+\kappa)-6 \delta^{2}(-4+5 \kappa)+\delta^{3}(-4+8 \kappa)\right\} \\
& /\left\{60+\delta^{2}(9-30 \kappa)+8 \delta^{3} \kappa+15 \delta(-3+2 \kappa)\right\}
\end{aligned}
$$

Figure 14 shows the variation of $\Omega$, as is calculated from Eq. (29) with the dimensionless thickness of the shell, $\delta$ at various parameters $\mathcal{K}$ (surface dilatational viscosity). It shows that $\Omega$ decreases as the thickness of the shell increases. This decrease is more pronounced at low values of the parameter $\kappa$ that is at lower surface dilatational viscosity of the surfactant layer. At $\delta=0$ there is no shell and hence, $\Omega=1$. As the thickness of the shell increases the drag force exerted to the particle decreases.

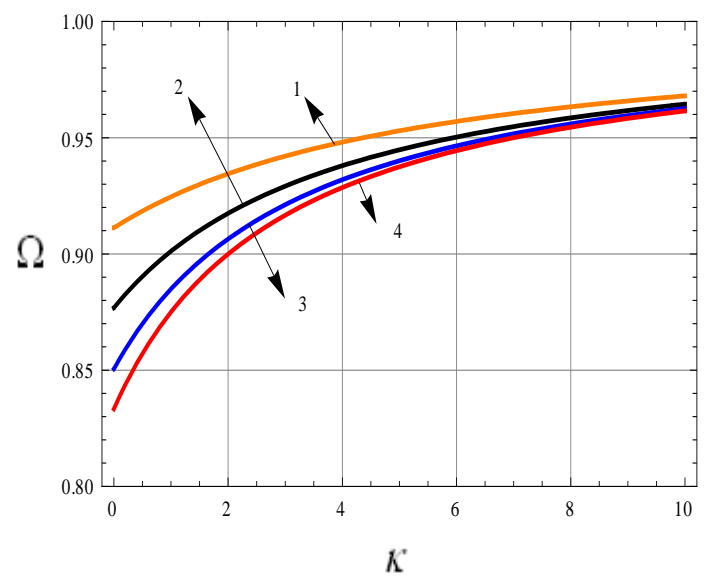

Fig. 12. Variation of $\Omega$ with surfactant layer parameter $\kappa$ at $\quad m=1 \quad$ and $\delta=0.4(1), \quad \delta=0.6(2)$, $\delta=0.8(3)$ and $\delta=1(4)$.

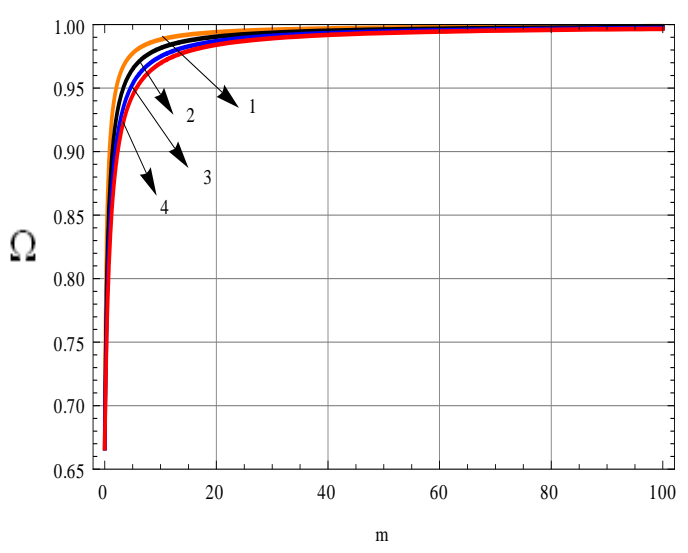

Fig. 13. Variation of $\Omega$ with $m$ (viscosity ratio) $\kappa=0$ $\delta=0.4(1), \delta=0.6(2), \delta=0.8$ (3) and $\delta=1(4)$.

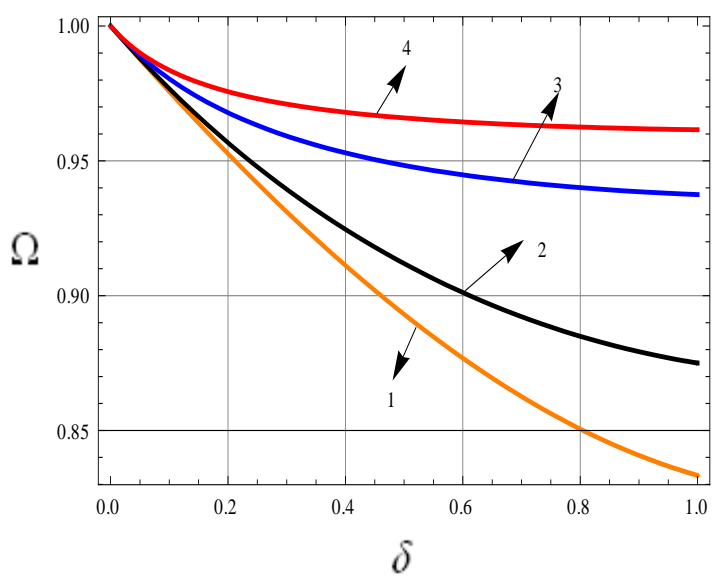

Fig. 14. Variation of $\Omega$ with the dimensionless thickness of the shell $\delta$ at $m=1$ and $\kappa=0(1), \kappa=1(2)$, $\kappa=5(3)$ and $\kappa=10(4)$ 


\section{REFERENCES}

Blawzdziewicz J., E .Vajnryb and M. Loewenberg (1999). Hydrodynamic interactions and collision efficiencies of spherical drops covered with an incompressible surfactant film. J.Fluid Mech.395, 29.

Blawzdziewicz J., P. Vlahovska and M. Loewenberg (2000). Rheology of a dilute emulsion of surfactantcovered spherical drops. Physica A 276, 50 -85.

Boussinesq, J. (1913). Existence of Surface Viscosity in the thin transition Layer Separating a Liquid from a Contiguous Fluid. Ann. chim. phys. 29, 349-357.

Cunningham, E. (1910). On the steady state fall of spherical particles through fluid medium. Proc $R$ Soc Lond; A, 83:357.

Danov, K.D (2001). On the Viscosity of dilute emulsions. Journal of Colloid and Interface Science 235,144-149.

Datta, S. and N.Pandya (2002). Deformation of the Drop with Surfactant Layer. Journal of International Academy of Physical Sciences 6, 9-19.

Gupta, B.R and S. Deo (2013). Axisymmetric creeping flow of a micropolar fluid over a sphere coated with a thin fluid film. J. of Applied Fluid Mechanics, 6, 2, 149-155.

Hadmard, J (1911). Mouvement permanent lent dune sphere liquid visquese dans un liquid visqueux. Compt. Rend. Acad. Sci. Paris Ser.A-B.152, 17351739.

Happel, J. (1958). Viscous flow in multiparticle systems: Slow motion of fluid relative to beds of spherical particles. J. A. I. Ch. E., 1958; 4, No.-2,197-201

Happel, J. (1959). Viscous flow relative to arrays of cylinders. A.I.Ch. E, 5(2), 174-177.

Happel, J. and H.Brenner (1983). Low Reynolds Number Hydrodynamics , Martinus Nijoff, The Hague, Netherlands.

Harper, J.F. (1974). On spherical bubble rising steadily in dilute surfactant solutions. Quart.J Mech. Appl Math 27, 87-100.

Harper, J.F. (1982). Surface activity and bubble motion. Appl.Sci.Res 38, 343-352.

Harper,J.F.(1988). The rear stagnation region of a bubble rising steadily in a dilute surfactant solution. Quart.J.Mech.Appl.Math.No.2, 41,203-213.

Kuwabara, S. (1959). The forces experienced by randomly distributed parallel circular cylinders or spheres in a viscous flow at small Reynolds number. J. Phys. Soc. Japan; 14, 527-532.

Kvashnin, A.G (1979). Cell model of suspension of spherical particles. Fluid Dynamics, 14,598- 602.

Lamb, H (1932). Hydrodynamics, $6^{\text {th }}$ edn. Cambridge University Press.

Levan, M.D. (1981). Motion of a droplet with a Newtonian interface. J.Colloid Interface Sci.83, 11.

Levan, M.D and Newman (1976). Effect of surfactant on terminal and interfacial velocities of bubble or drop. $J$. AIChE J. 22, 695.

Li, X.J and C. Pozrikidis (1997). Effects of surfactants on drop deformation and on the rheology of dilute emulsions in Stokes flow. J.Fluid Mech.341, 165194.

Li, X.J and Z.S Mao (2001). The Effect of surfactant on the motion of a buoyancy-driven drop at intermediate Reynolds nos: A numerical approach. J. Colloid Interface Sci. 240, 307-322.

Masliyah, J., G. Neale and K Malysa and T.G.M Van de Ven (1987). Creeping flow over a composite sphere: Solid core with porous shell, Chem. Eng. Sci.42, 245253.

McLaughlin, J. B. (1996). Numerical simulation of bubble motion in water. Journal of Colloid and Interface Science, 184, 614-625.

Mehta, G.D. and T.F Morse (1975). Flow through charged membranes. J. Chem. Phys, 63 (5), 1878-1889.

Miller, C.A and L.E Scriven (1968). The oscillation of a liquid droplet immersed in another fluid. J Fluid Mech. 32, 417, 126, 237-250.

Prosperetti, A. (1977). Viscous effects on perturbed spherical flows. Q. Appl.Math, 35, 339.

Prosperetti, A. (1980). Free oscillations of drops and bubbles: the initial-value problem. J Fluid Mech., 100,333 .

Ramabhadran, R. E., C. H. Byers, and J. C. Friedly (1976). On the Dynamics of Fluid interfaces. A.I.Ch.E.J., 22, 872.

Reid, W.H. (1960). The oscillations of a viscous liquid drop. Q.Appl.Maths 18, 88.

Rybczynski, W. (1911). Uber die fortschreitende Bewegung einer flussigen Kugel in einem zahen medium. Bull. Int. Acad. Sci. Cracovie. A., 40-46.

Ryskin, G. and L. G. Leal (1983). Orthogonal mapping. Journal of Computational Physics, 50, 71-100. 
S. Datta and S. Raturi. / JAFM, Vol. 7, No. 2, pp. 263-273, 2014.

Ryskin, G. and L.G. Leal (1984a). Numerical solution of free-boundary problems in fluid mechanics. Part 1.

Ryskin, G. and L.G. Leal (1984b). Numerical solution of free-boundary problems in fluid mechanics. Part 2.Buoyancy-driven motion of a gas bubble through a quiescent liquid. J. of Fluid Mechanics, 148, 19-35.

Sadhal, S.S and R.E. Johnson (1983). Stokes flow past bubbles and drops partially coated with thin films. Part I. Stagnant cap of surfactant film-exact solution. J. of Fluid Mechanics, 126.237-250.

Scriven, L.E. (1960). Dynamics of a fluid interface: Equation of motion for Newtonian surface fluids. Chem. Eng. Sci. 12, 98-108.

Stone, H.A. and L.G. Leal (1990). The effect of surfactants on drop deformation and breakup. J. of Fluid Mech. $220,161-186$.
Stone, H.A. (1994). Dynamics of drop deformation and breakup in viscous fluid. Ann. Rev. Fluid Mech. 26, 65-102.

Taylor, T.D. and A. Acrivos (1964). On the deformation and drag of a falling viscous drop at low Reynolds number. J. Fluid Mech. 18, 466-476.

Uchida, S. (1949). Viscous Flow in Multiparticle Systems: Slow Viscous Flow Through a Mass of Particles, Int. Sci. Technol. Univ. Tokyo (in Japanese) 397 Abstract, Ind. Eng. Chem. 46 (1954) 1194-1195 (translated by T. Motai).

Vasin, S.I., A.N Filippov and V.M. Starov (2008). Hydrodynamic permeability of membranes built up by particles covered by porous shells: Cell models. Advances in Colloid and Interface Science 139, 83-96.

Warszynski, P., B.Jachimska and K. Malysa (1996) Experimental evidence of the existence of nonequilibrium coverages over the surface of the floating bubble. Colloids and Surfaces A: Physicochemical Engineering Aspects, 108, 321-325 